\title{
Antimicrobial Activity and Protease Stability of Peptides Containing Fluorinated Amino Acids
}

$\mathrm{He}^{\mathrm{Meng}}{ }^{\dagger}$ and Krishna Kumar ${ }^{\dagger, \S, *}$

${ }^{\dagger}$ Department of Chemistry, Tufts University, Medford, MA 02155 and ${ }^{\S}$ Cancer Center, Tufts-New England Medical Center, Boston, MA 02110

Supporting Information

\section{Protease Cleavage Patterns}

HPLC traces of digestive mixtures at various time intervals are plotted with offset abscissae to facilitate visualization. Traces for BII1, BII1F2, BII5, BII5F2 are shown in Fig. S1; BII6, BII6F2, BII10, BII10F2 in Fig. S2; and M2, M2F2 in Fig. S3. Each peak was identified by ESI-MS (identities shown in the supplementary tables). The cleavage patterns are altered due to the presence of hexafluoroleucine at $\mathrm{P} 1$ ' or/and $\mathrm{P} 2$ ' position.
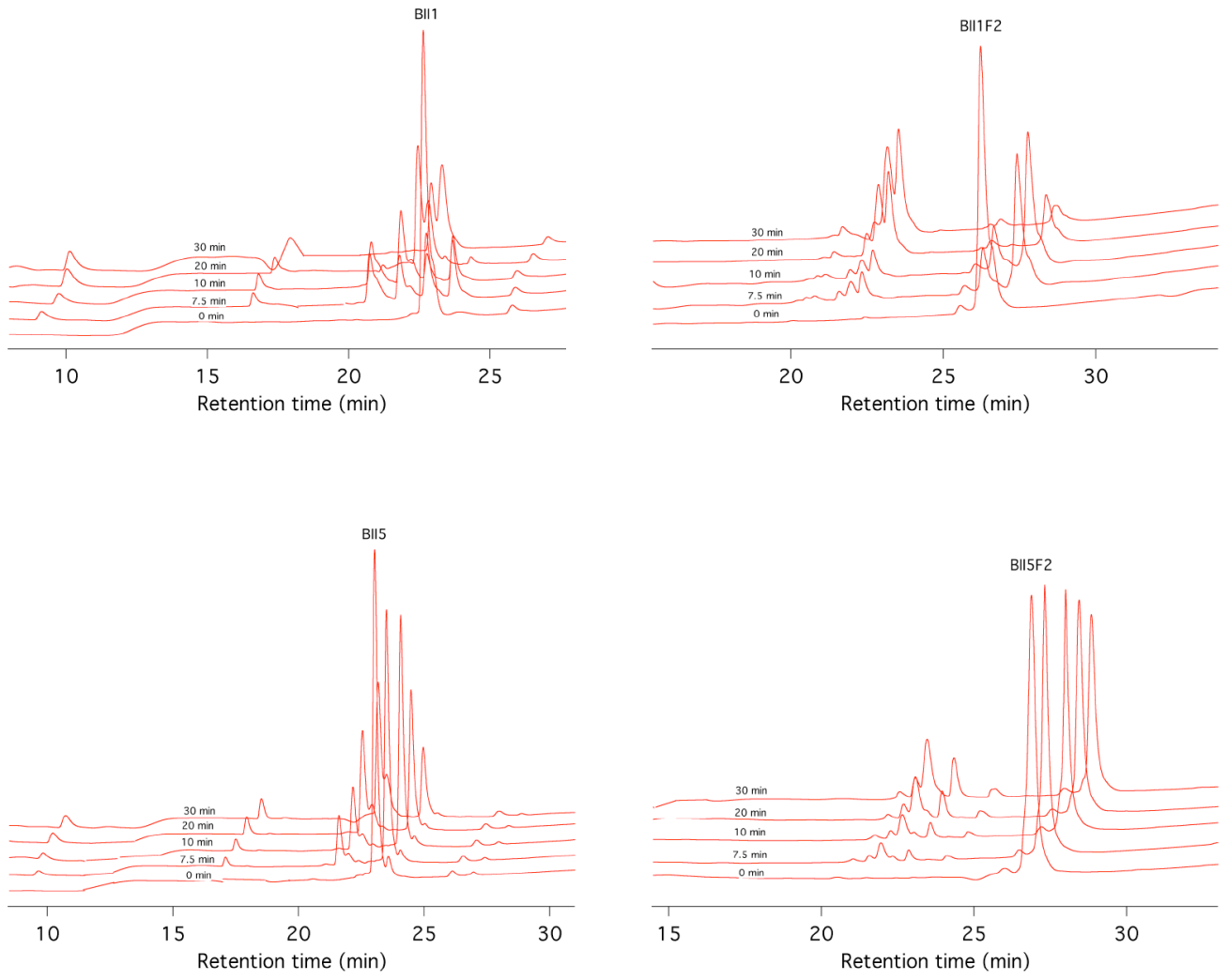

Figure S1. HPLC analysis of trypsin digestion mixtures of BII1, BII1F2, BII5, and BII5F2. The peptides being proteolyzed are labeled in each graph. Only HPLC traces at $t=0,7.5$, 10, 20, $30 \mathrm{~min}$ are shown. 

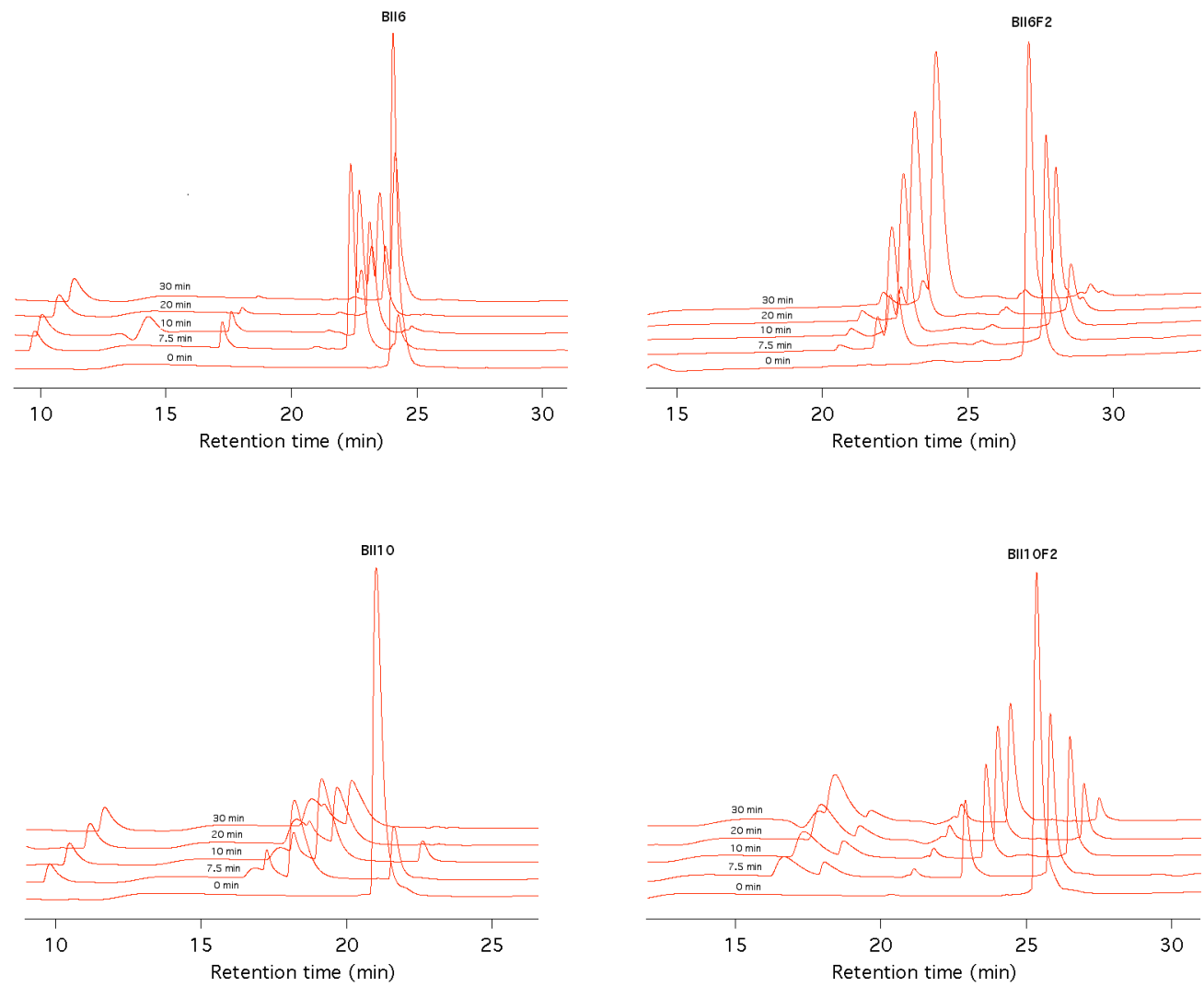

Figure S2. HPLC analysis of trypsin digestion mixtures of BII6, BII6F2, BII10, and BII10F2. The peptides being proteolyzed are labeled in each graph. Only HPLC traces at $t=0,7.5,10,20$, 30 min were plotted.
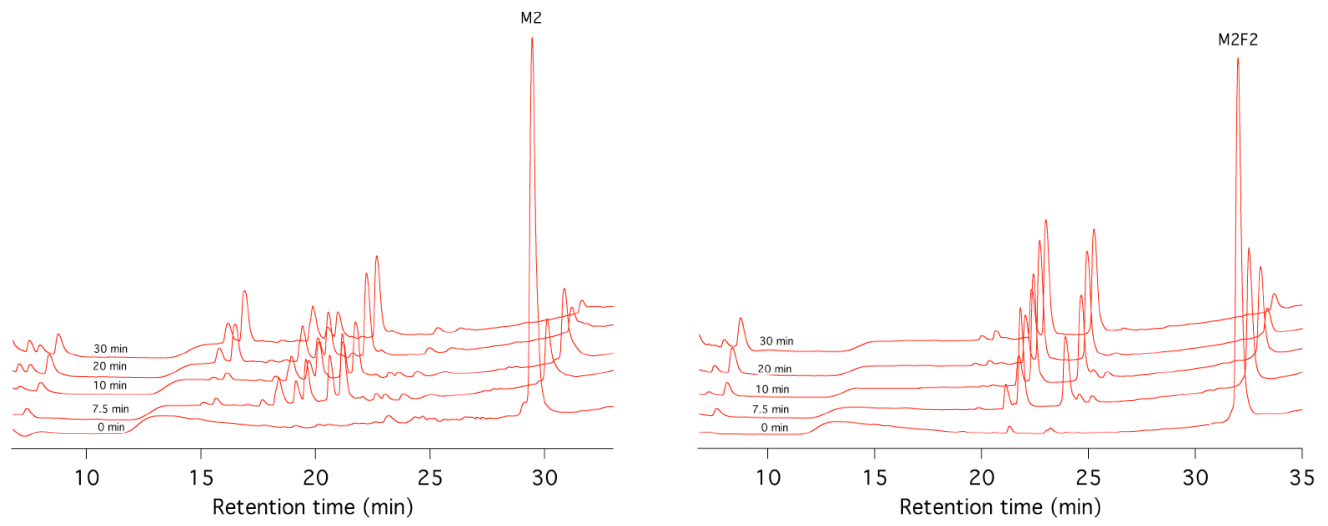

Figure S3. HPLC analysis of trypsin digestion mixtures of M2 and M2F2. 


\section{Tables for Identification of Peptide Fragments in Protease Stability}

Fractions at different retention times were collected (J.T.Baker $\mathrm{C}_{18}, 5 \mu \mathrm{m}, 4 \mathrm{~mm} \times 250$ $\mathrm{mm}$ ) and were directly analyzed by ESI-MS.

Table S1. Identification of proteolyzed fragments of BII1 by ESI-MS

\begin{tabular}{ccccc}
\hline Ret time $(\min )$ & Name & Fragment & MW $_{\text {calcd }}(\mathrm{Da})$ & $\mathrm{MW}_{\text {obsd }}(\mathrm{Da})$ \\
\hline 9.4 & BII1(18-21) & LLRK & 528.4 & 529.5 \\
20.7 & BII1(1-17) & TRSSRAGLQFPVGRVHR & 1924.07 & 1925.0 \\
21.8 & BII1(6-17) & AGLQFPVGRVHR & 1336.76 & 1337.4 \\
22.7 & BII1(1-21) & TRSSRAGLQFPVGRVHRLLRK & 2434.44 & 2436.0 \\
23.7 & BII1(6-21) & AGLQFPVGRVHRLLRK & 1847.12 & 1848.0 \\
\hline
\end{tabular}

Table S2. Identification of proteolyzed fragments of BII1F2 by ESI-MS

\begin{tabular}{ccccc}
\hline Ret time $(\mathrm{min})$ & Name & Fragment & $\mathrm{MW}_{\text {calcd }}(\mathrm{Da})$ & $\mathrm{MW}_{\text {obsd }}(\mathrm{Da})$ \\
\hline 20.8 & Bll1F2(18-21) & LLRK & 744.37 & 745.28 \\
21.9 & Bll1F2(6-17) & AGLQFPVGRVHR & 1336.76 & 1337.4 \\
22.3 & Bll1F2(6-14) & AGLQFPVGR & 943.52 & 944.5 \\
22.6 & Bll1F2(15-21) & VHRLLRK & 1136.48 & 1137.8 \\
26.2 & BII1F2(1-21) & TRSSRAGLQFPVGRVHRLLRK & 2650.62 & 2651.0 \\
27.5 & BII1F2(6-21) & AGLQFPVGRVHRLLRK & 2063.01 & 2064.0 \\
\hline
\end{tabular}

* L: hexafluoroleucine.

Table S3. Identification of proteolyzed fragments of BII5 by ESI-MS

\begin{tabular}{ccccc}
\hline Ret time $(\min )$ & Name & Fragment & MW $_{\text {calcd }}(\mathrm{Da})$ & $\mathrm{MW}_{\text {obsd }}(\mathrm{Da})$ \\
\hline 9.4 & BII5(18-21) & LLRK & 528.37 & 529.49 \\
17.0 & BII5(15-21) & VHRLLRK & 920.60 & 921.80 \\
21.0 & BII5(5-17) & RAGLQFPVGRVHR & 1491.85 & 1493.42 \\
22.0 & BII5(5-14) & RAGLQFPVGR & 1099.63 & 1101.00 \\
23.0 & BII5(5-21) & RAGLQFPVGRVHRLLRK & 2003.43 & 2004.00 \\
\hline
\end{tabular}

Table S4. Identification of proteolyzed fragments of BII5F2 by ESI-MS

\begin{tabular}{ccccc}
\hline Ret time $(\min )$ & Name & Fragment & MW $_{\text {calcd }}(\mathrm{Da})$ & $\mathrm{MW}_{\text {obsd }}(\mathrm{Da})$ \\
\hline 20.8 & BlI5F2(18-21) & LLRK & 744.37 & 745.28 \\
21.2 & BlI5F2(5-17) & RAGLQFPVGRVHR & 1491.85 & 1493.32 \\
21.8 & BII5F2(5-14) & RAGLQFPVGR & 1099.63 & 1100.92 \\
22.6 & BII5F2(15-21) & VHRLLRK & 1136.00 & 1137.82 \\
27.0 & BII5F2(5-21) & RAGLQFPVGRVHRLLRK & 2219.17 & 2220.45 \\
\hline
\end{tabular}

Table S5. Identification of proteolyzed fragments of BII6 by ESI-MS

\begin{tabular}{ccccc}
\hline Ret time $(\mathrm{min})$ & Name & Fragment & MW $_{\text {calcd }}(\mathrm{Da})$ & $\mathrm{MW}_{\text {obsd }}(\mathrm{Da})$ \\
\hline 9.4 & Bll6(18-21) & LLRK & 528.37 & 529.49 \\
17.0 & Bll6(15-21) & VHRLLRK & 920.60 & 921.80 \\
22.0 & Bll6(6-17) & AGLQFPVGRVHR & 1335.57 & 1336.82 \\
22.4 & Bll6(6-14) & AGLQFPVGR & 943.52 & 944.45 \\
23.6 & Bll5(6-21) & AGLQFPVGRVHRLLRK & 1847.24 & 1848.73 \\
\hline
\end{tabular}

Table S6. Identification of proteolyzed fragments of BII6F2 by ESI-MS

\begin{tabular}{ccccc}
\hline Ret time $(\mathrm{min})$ & Name & Fragment & MW $_{\text {calcd }}(\mathrm{Da})$ & $\mathrm{MW}_{\text {obsd }}(\mathrm{Da})$ \\
\hline 20.8 & Bll6F2(18-21) & LLRK & 744.37 & 745.28 \\
21.9 & Bll6F2(6-17) & AGLQFPVGRVHR & 1336.76 & 1337.4 \\
22.3 & Bll6F2(6-14) & AGLQFPVGR & 943.52 & 944.5 \\
22.6 & Bll6F2(15-21) & VHRLLRK & 1136.48 & 1137.8 \\
27.4 & Bll6F2(6-21) & AGLQFPVGRVHRLLRK & 2063.13 & 2064.32 \\
\hline
\end{tabular}


Table S7. Identification of proteolyzed fragments of BII10 by ESI-MS

\begin{tabular}{ccccc}
\hline Ret time $(\mathrm{min})$ & Name & Fragment & MW $_{\text {calcd }}(\mathrm{Da})$ & MW $_{\text {obsd }}(\mathrm{Da})$ \\
\hline 9.4 & BII10(18-21) & LLRK & 528.37 & 529.49 \\
16.4 & BII10(10-14) & FPVGR & 574.32 & 575.36 \\
17.5 & BII10(10-17) & FPVGRVHR & 966.56 & 967.72 \\
23.0 & BII10(10-21) & RAGLQFPVGRVHRLRK & 1477.82 & 1479.18 \\
\hline
\end{tabular}

Table S8. Identification of proteolyzed fragments of BII10F2 by ESI-MS

\begin{tabular}{ccccc}
\hline Ret time $(\mathrm{min})$ & Name & Fragment & MW $_{\text {calcd }}(\mathrm{Da})$ & $\mathrm{MW}_{\text {obsd }}(\mathrm{Da})$ \\
\hline 16.3 & Bll10F2(10-14) & FPVGR & 574.32 & 575.36 \\
17.3 & Bll10F2(10-17) & FPVGRVHR & 966.55 & 976.54 \\
20.8 & Bll10F2(18-21) & LLRK & 744.37 & 745.28 \\
22.6 & Bll10F2(15-21) & VHRLLRK & 1136.00 & 1137.54 \\
25.5 & Bll10F2(10-21) & FPVGRVHRLLRK & 1693.70 & 1694.28 \\
\hline
\end{tabular}

Table S9. Identification of proteolyzed fragments of M2 by ESI-MS.

\begin{tabular}{|c|c|c|c|c|}
\hline $\begin{array}{l}\text { Ret time } \\
(\min )\end{array}$ & Name & Fragment & $\mathrm{MW}_{\text {calcd }}(\mathrm{Da})$ & $\mathrm{MW}_{\text {obsd }}(\mathrm{Da})$ \\
\hline 6.7 & M2(11-14) & KFAK & 492.62 & 493.63 \\
\hline 14.2 & $\mathrm{M} 2(1-4)$ & GIGK & 373.23 & $399.06(+\mathrm{Na})$ \\
\hline 14.8 & M2(5-10) & FLHAAK & 685.39 & 686.45 \\
\hline 17.5 & M2(12-20) & FAKAFVAEI & 994.55 & 996.34 \\
\hline 18.5 & $M 2(1-11)$ & GIGKFLHAAKK & 1168.71 & 1170.06 \\
\hline 18.8 & M2(1-10) & GIGKFLHAAK & 1041.61 & 1042.18 \\
\hline 20.2 & M2(1-14) & GIGKFLHAAKKFAK & 1514.91 & 1516.05 \\
\hline 20.8 & M2(15-23) & AFVAEIMNS & 979.48 & 980.34 \\
\hline 29.6 & M2(1-23) & GIGKFLHAAKKFAKAFVAEIMNS & 2476.38 & 2477.45 \\
\hline
\end{tabular}

Table S10. Identification of proteolyzed fragments of M2F2 by ESI-MS.

\begin{tabular}{ccccc}
\hline Ret time $(\min )$ & Name & Fragment & MW $_{\text {calcd }}(\mathrm{Da})$ & $\mathrm{MW}_{\text {obsd }}(\mathrm{Da})$ \\
\hline 6.7 & M2F2(11-14) & KFAK & 492.62 & 493.41 \\
17.8 & M2F2(5-11) & FLHAAKK & 921.49 & 922.51 \\
18.4 & M2F2(5-10) & FLHAAK & 793.39 & 794.46 \\
20.1 & M2F2(1-11) & GIGKFLHAAKK & 1276.71 & 1277.74 \\
20.8 & M2F2(1-14) & GIGKFLHAAKKFAK & 1622.91 & 1625.42 \\
23.1 & M2F2(15-23) & AFVAELMNS & 1087.48 & 1088.49 \\
32.0 & M2F2(1-23) & GIGKFLHAAKKFAKAFVAELMNS & 2692.26 & 2693.74 \\
\hline
\end{tabular}

Table S11. Initial pseudo first order rate constants for protease degradation reactions.

\begin{tabular}{cc}
\hline Peptides & Initial rate constants $\left(\mathrm{min}^{-1}\right)$ \\
\hline BII1 & $0.203 \pm 0.005$ \\
BII1F2 & $0.230 \pm 0.016$ \\
BII5 & $0.095 \pm 0.005$ \\
BII5F2 & $0.043 \pm 0.003$ \\
BII6 & $0.231 \pm 0.015$ \\
BII6F2 & $0.102 \pm 0.005$ \\
Bll10 & $0.195 \pm 0.024$ \\
BII10F2 & $0.089 \pm 0.077$ \\
\hline M2 & $0.232 \pm 0.020$ \\
M2F2 & $0.173 \pm 0.055$ \\
M2F5 & $1.82 \times 10^{-3} \pm 0.22 \times 10^{-3}$ \\
\hline
\end{tabular}




\section{Hydrolysis of M2F5 by Trypsin}

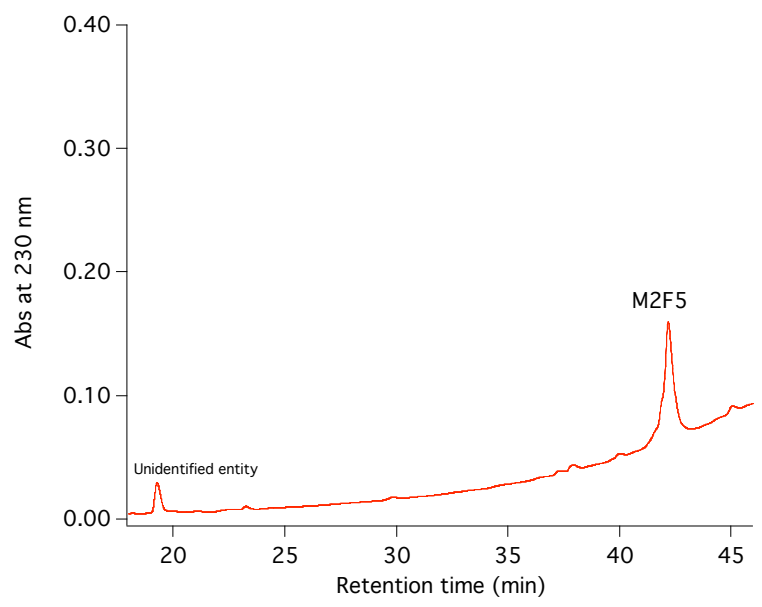

Figure S4. HPLC trace of reaction mixture after $24 \mathrm{~h}$ incubation of M2F5 with trypsin at $37^{\circ} \mathrm{C}$. No decrease in the peak corresponding to M2F5 was seen during this time. 


\section{Data for TFE Titration - BII1, BII1F2, BII5, and BII5F2}

Peptides were titrated with increasing amounts of 2,2,2-trifluoroethanol (TFE) in PBS resulting in higher amounts of helical induction.

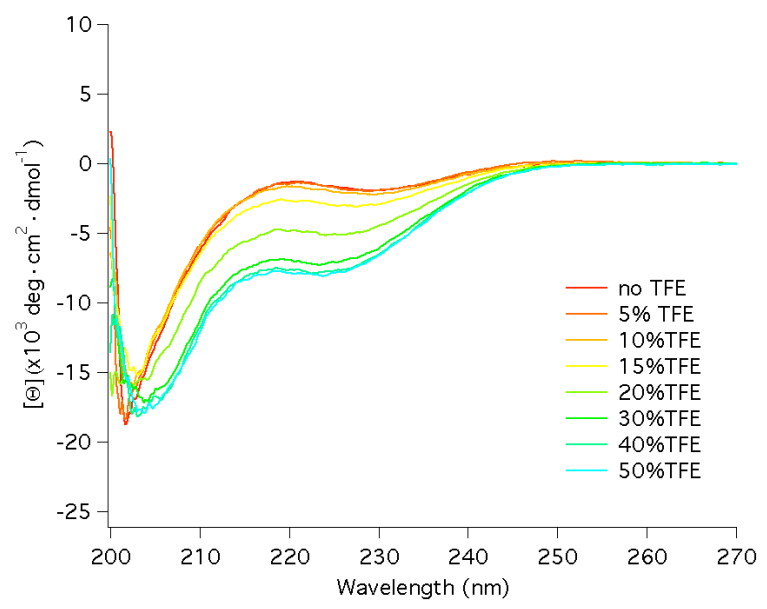

Figure S5. CD data of TFE titration (BII1).

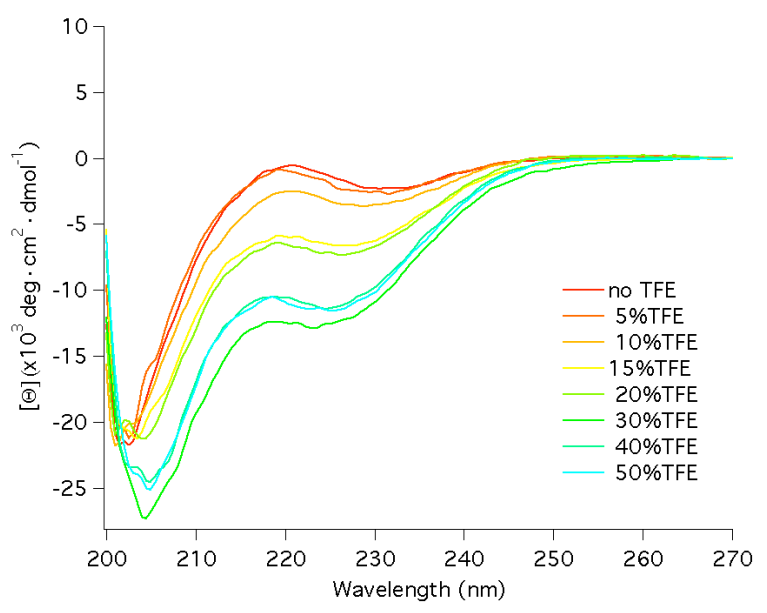

Figure S7. CD data of TFE titration (BII5).

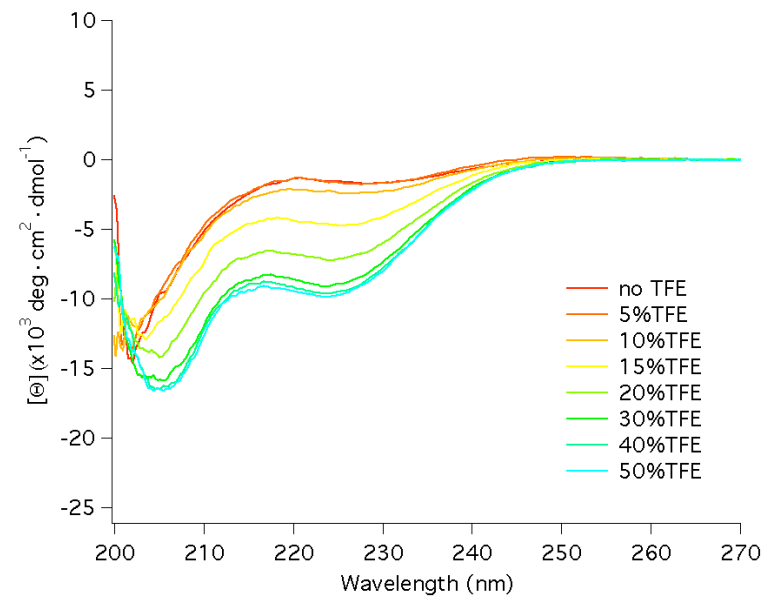

Figure S6. CD data of TFE titration (BII1F2).

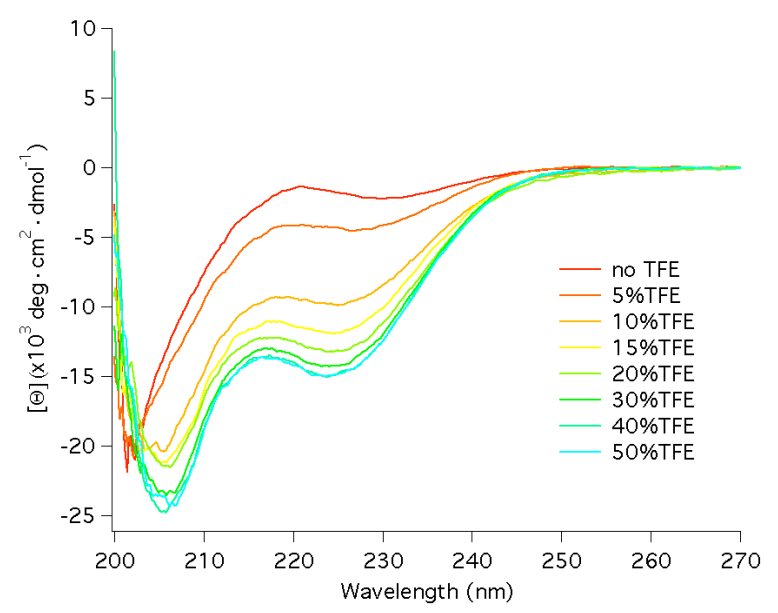

Figure S8. CD data of TFE titration (BII5F2). 


\section{Data for TFE Titration - BII6, BII6F2, BII10, and BII10F2}

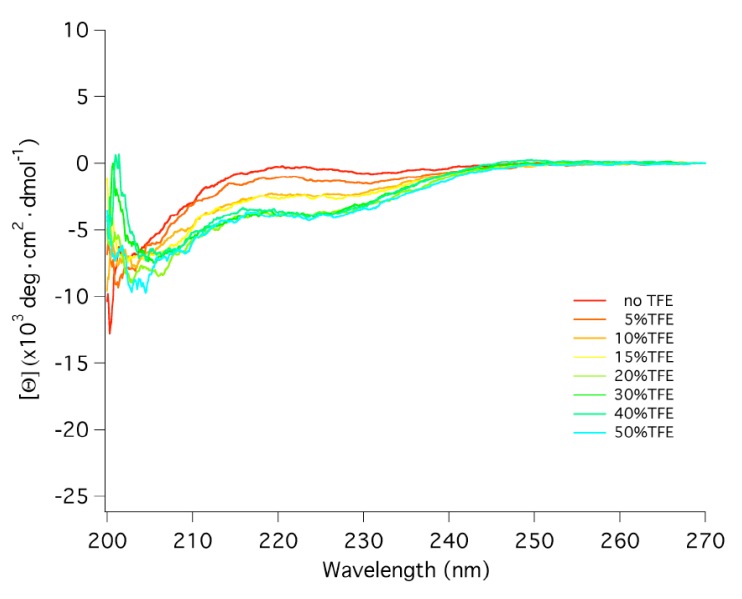

Figure S9. CD data of TFE titration (BII6).

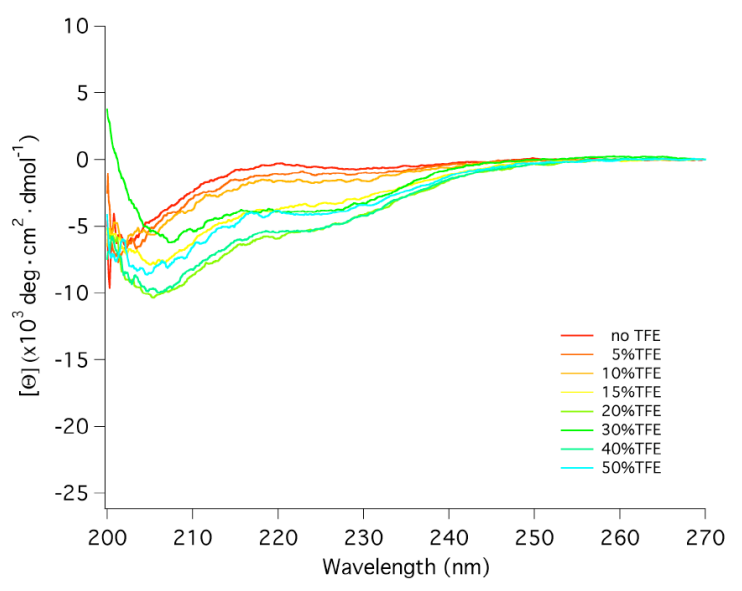

Figure S11. CD data of TFE titration (BII10).

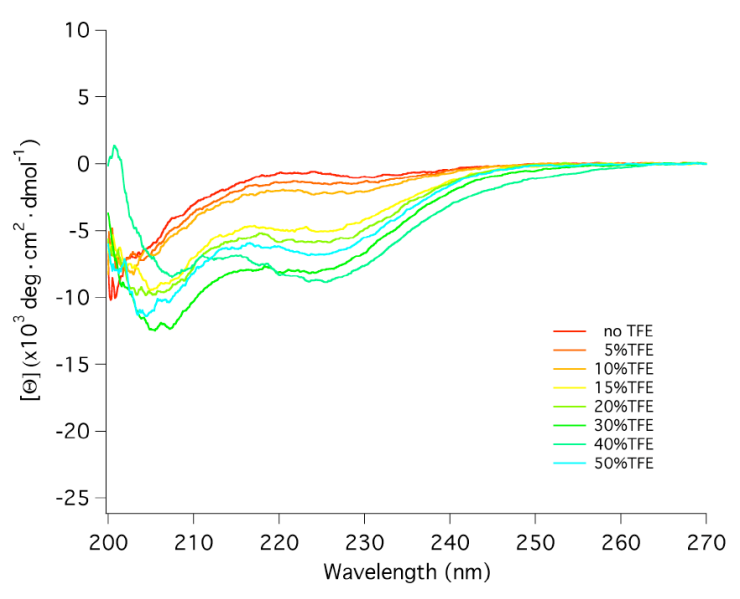

Figure S10. CD data of TFE titration (BII6F2).

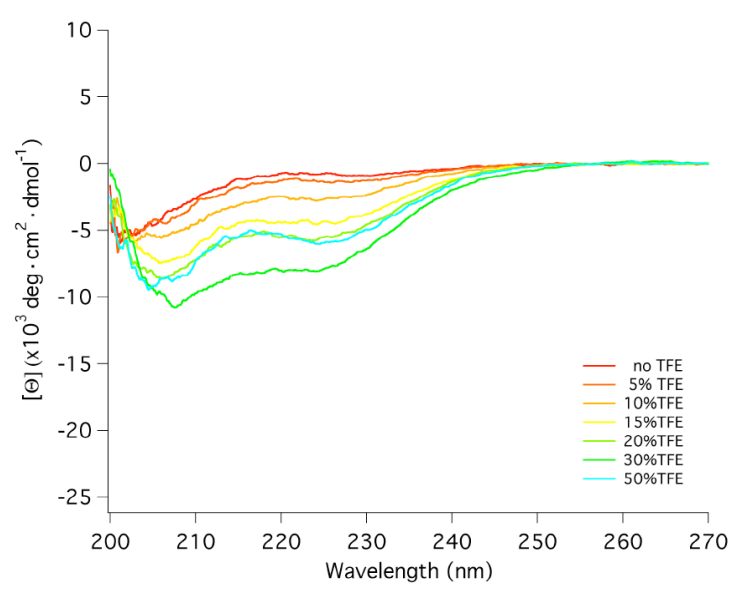

Figure S12. CD data of TFE titration (BII10F2). 
CD Data for TFE Titration - M2, M2F2, and M2F5

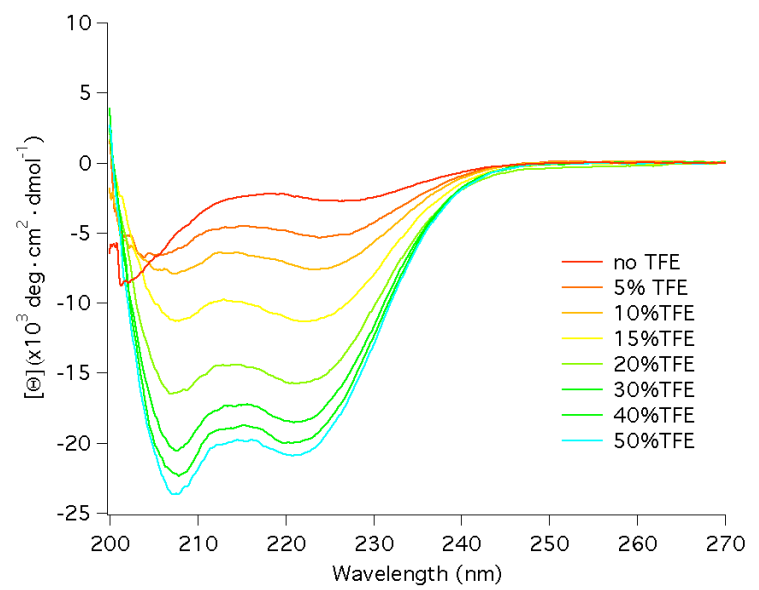

Figure S13. CD data of TFE titration (M2).

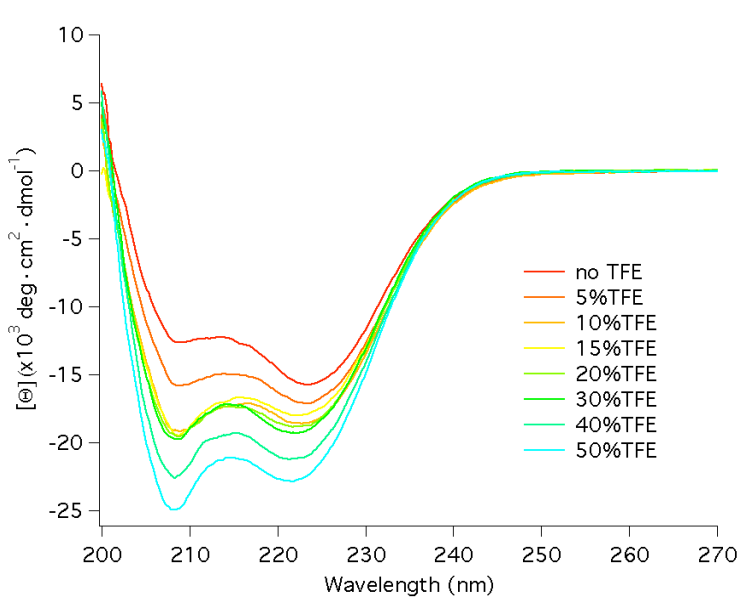

Figure S15. CD data of TFE titration (M2F5).

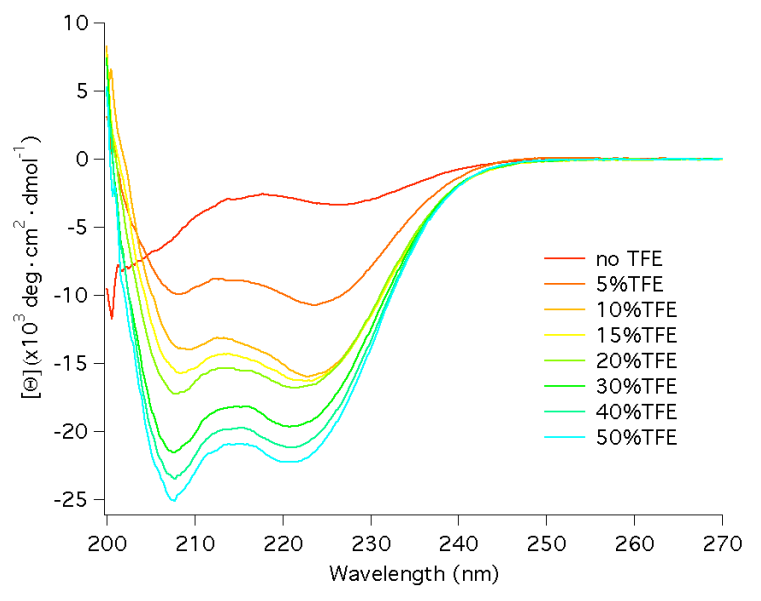

Figure S14. CD data of TFE titration (M2F2).

\section{Analytical Ultracentrifugation}

Table S12. Apparent molecular weights determined by equilibrium sedimentation.

$$
\mathrm{MW}_{\text {approx }} \text { (no. of helices) }
$$

\begin{tabular}{ccc} 
Peptide & $50 \mu \mathrm{M}$ & $100 \mu \mathrm{M}$ \\
\hline M2 & $2478(0.99)$ & $2413(0.97)$ \\
M2F2 $^{*}$ & $12401(4.60)$ & $12685(4.71)$ \\
M2F5 $^{*}$ & $12431(3.99)$ & $14420(4.63)$ \\
\hline
\end{tabular}

All samples were run in $10 \mathrm{mM}$ phosphate $\mathrm{pH} 7.4,137 \mathrm{mM} \mathrm{NaCl}, 2.7 \mathrm{mM} \mathrm{KCl} .{ }^{*}$ Fits to single ideal species model gave non-random residuals. 